\title{
Enquête
}

Archives de la revue Enquête

5 | 1997

Débats et controverses

\section{La querelle savante dans l'Europe de la Renaissance}

Éthique et étiquette

The scholarly quarrel in Renaissance Europe. Ethics and Etiquette

Isabelle Pantin

\section{CpenEdition}

Journals

Édition électronique

URL : http://journals.openedition.org/enquete/1093

DOI : 10.4000/enquete. 1093

ISSN : 1953-809X

Éditeur :

Cercom, Éditions Parenthèses

Édition imprimée

Date de publication : 1 septembre 1997

Pagination : 71-82

Référence électronique

Isabelle Pantin, «La querelle savante dans l'Europe de la Renaissance », Enquête [En ligne], 5 | 1997,

mis en ligne le 15 juillet 2013, consulté le 20 mars 2020. URL : http://journals.openedition.org/

enquete/1093 ; DOI : https://doi.org/10.4000/enquete.1093

Ce document a été généré automatiquement le 20 mars 2020. 


\section{La querelle savante dans l'Europe de la Renaissance}

Éthique et étiquette

The scholarly quarrel in Renaissance Europe. Ethics and Etiquette

\section{Isabelle Pantin}

1 La querelle savante a-t-elle jamais ressemblé, de près ou de loin, à un sport international, pour risquer une analogie avec ce qu'était devenu le tournoi aux XIV et $\mathrm{XV}^{\mathrm{e}}$ siècles ou le jeu de paume au $\mathrm{XVI}^{\mathrm{e} 1}$ ? Cette question est sûrement paradoxale, peutêtre oiseuse, mais elle peut servir de point de départ à une enquête sur la sociabilité et le savoir-vivre des intellectuels européens, dans un domaine important de leur activité.

2 Le débat par la parole directe ou écrite était, à notre connaissance, partie intégrante de la vie scientifique depuis les Grecs, même s'il ne jouissait pas d'un prestige égal à celui qui était réservé à la spéculation contemplative. Au Moyen Âge il s'épanouit sous diverses formes, universitaires, courtoises, plus ou moins réglementées. Or le développement de l'imprimerie lui ouvre des possibilités supplémentaires: il lui confère une plus longue portée, en offrant l'occasion de se mesurer à des partenaires éloignés, parfois étrangers, inconnus l'un à l'autre; et il achève de lui donner la dimension du public. Le match ou la joute, si l'on veut reprendre les analogies initiales, prend plus de sens pour se dérouler au su d'une multiplicité indéfinie de témoins virtuels; une querelle privée peut ainsi acquérir au plus haut point une qualité que les débats académiques ne possèdent pas toujours, celle d'un spectacle exemplaire.

Parmi la grande variété des conflits advenus en milieu savant, j'ai donc retenu le petit échantillon des querelles qui sont passées par le canal du livre imprimé : pour les raisons qui viennent d'être évoquées, et aussi parce que leurs traces sont le mieux conservées. Qui voudra faire l'histoire de la disputatio à la Renaissance devra se contenter de textes théoriques, de règlements universitaires et de ces livrets squelettiques que sont les positions de thèses; tandis que nous connaissons les principales péripéties et l'essentiel des débats de l'affaire du Ciceronianus, dans lequel Érasme attaquait le respect idolâtre du style de Cicéron pour avancer un exemple 
illustre. Je n'ai pas tenté de dresser un inventaire exhaustif, ni d'esquisser la moindre statistique, par discipline, par pays, etc. Je me suis limitée à quelques cas, en excluant le domaine théologique mais, cette réserve faite, en faisant abstraction $d u$ fond: l'important n'était pas d'analyser l'objet des discussions mais d'examiner les procédures. Ce choix étant fait, l'enquête s'est orientée vers la mise en évidence d'un savoir-vivre international de la querelle, fondé sur une sorte de philosophie homogène et sur la reconnaissance de valeurs éthiques communes, sans oublier pourtant de noter d'éventuels particularismes nationaux.

4 Tous les savants d'Europe avaient subi un dressage similaire qui les avait dotés des mêmes réflexes, du même bagage doctrinal et de la même façon de défendre leurs idées. Il ne m'appartient pas d'évoquer le rôle des universités, qui pouvaient former, d'un bout à l'autre de l'Europe, des intellectuels capables d'échanges, sinon interchangeables. Je soulignerai seulement la large extension de cette éducation commune : elle ne concernait pas seulement les plus savants docteurs, mais tous les jeunes bourgeois ou gentilshommes qui fréquentaient les collèges ; et j'insisterai sur sa longévité : malgré les soucis de réforme, une bonne part de l'héritage scolastique s'est transmis à la pédagogie des humanistes et, de là, à celle des jésuites. L'exemple de la disputatio le montre assez clairement.

5 Cette pratique destinée à prouver la capacité d'un étudiant à « défendre une position » avait été mise au point par les sophistes, mais ce fut sous l'autorité d'Aristote ${ }^{2}$ qu'elle fut adoptée dans les universités médiévales. Il s'agissait, pour simplifier, de débats sur une question donnée, entre deux adversaires qui se partageaient les rôles, sous le contrôle d'un arbitre (le moderator ou praeses) : l'un défendait la thèse, en répondant aux objections de l'autre qui s'efforçait de le faire tomber dans l'absurdité et les contradictions, ou de le réduire au silence, en lui posant des séries de questions habilement agencées. Si nous en croyons les descriptions horrifiées des humanistes, l'exercice avait dégénéré au point d'exprimer toute la brutalité d'une barbarie gothique. Au lieu de suivre honnêtement les règles, dans un esprit sportif et désintéressé, les adversaires avaient recours à la mauvaise foi, aux hurlements, aux injures, aux coups, et ils étaient surtout excités par l'espoir de gagner de l'argent ${ }^{3}$. Bref, l'antique disputatio était devenue une sorte de combat de coqs, honteusement intégré dans les activités académiques.

6 Toutefois les réformateurs les plus radicaux ne songèrent jamais à la supprimer ; il leur suffisait de mieux l'encadrer, de canaliser sa violence, de lui rendre son caractère originel de sport dialectique. Vivès, par exemple, souhaitait qu'elle fût réservée aux étudiants déjà bien endoctrinés au lieu de mettre aux prises de jeunes ignorants ${ }^{4}$; et cette idée fut reprise par Ramus, pour qui elle devait servir de moyen de récapitulation et de contrôle ${ }^{5}$, avant d'être appliquée dans les collèges jésuites ${ }^{6}$. La reconnaissance générale de l'utilité de la dispute s'ancrait dans une tradition éducative essentiellement orale, où la discussion passait pour le meilleur moyen d'éprouver les idées. De plus, elle reposait sur une anthropologie particulière. Les pédagogues ou les moralistes qui se mêlaient de la question montraient volontiers en l'homme un être perpétuellement menacé par l'engourdissement intellectuel, et qu'il convenait de réveiller en l'engageant dans des combats revigorants : au savant mélancolique et solitaire, prêt à tomber des abîmes de la spéculation dans les gouffres de l'acedia, devait s'opposer l'alerte controversiste.

Les disputes furent instituées jadis, mais entre les jeunes gens, pour exciter cette vigueur de l'esprit, souvent plongée dans la torpeur, pour qu'ils soient plus vifs à 
l'étude et que, soit qu'ils vainquent, soit qu'ils évitent d'être vaincus, ils enfoncent plus profondément « dans leur tête » les leçons de leurs maîtres ${ }^{7}$.

Vivès soutenait cette idée au début du siècle, mais on citerait aussi bien des textes beaucoup plus tardifs, comme cet extrait de la Ratio studiorum de 1586 :

Quand l'adversaire nous presse, nous sommes contraints de tendre toutes nos facultés, alors naissent, jaillissent sous les instances d'attaquants, une foule d'idées qui ne nous seraient jamais venues dans la quiétude du cabinet [litt.: dans la quiétude et l'ombre] $]^{8}$.

Ou encore Montaigne :

L'estude des livres, c'est un mouvement languissant et foible qui n'eschauffe poinct : là où la conference apprend et exerce en un coup. Si je confere avec une ame forte et un roide jousteur, il me presse les flancs, me pique à gauche et à dextre, ses imaginations eslancent les miennes. La jalousie, la gloire, la contention me poussent et rehaussent au-dessus de moy-mesmes ${ }^{9}$.

Ce fut aussi dans le même esprit que Scaliger baptisa Exercitationes le gros volume qu'il publia en 1557 pour réfuter le De subtilitate où Cardan exposait certaines des thèses de sa nouvelle physique. Et les nombreux liminaires qui figuraient dans l'ouvrage attestaient du caractère sportif de l'entreprise : l'attaque était un hommage puisqu'elle prouvait la fécondité d'un texte capable de susciter une aussi belle activité critique ${ }^{10}$. Dans l'Excuse finale, Scaliger priait d'ailleurs Cardan de prendre l'assaut en bonne part ; le combat comportait en lui-même son profit, sans viser l'humiliation d'un des partenaires : ils s'escrimaient à égalité puisque les mêmes ténèbres d'ignorance les environnaient tous deux ${ }^{11}$.

9 Un autre élément propre à situer les querelles savantes sur un fond éthique homogène, était leur participation au système général de l'honneur, ce qui pose la question générale des rapports entre le duel armé et la controverse verbale. À la Renaissance, les règles de savoir-vivre et les normes de comportement, valables sur différents plans sinon en toutes circonstances, se référaient, du moins pour leurs théoriciens, à un manuel d'audience européenne, l'Éthique a Nicomaque. Celui-ci fixait pour la plupart des états, à l'exclusion des gens mécaniques ${ }^{12}$, les contours de notions aussi essentielles que la vertu, l'honneur, la magnanimité, l'attitude à adopter face à l'injure ${ }^{13}$. De façon significative, le Dialogo dell'honore de Giovanni Battista Possevino, farci de citations aristotéliciennes, montre à la fois les similitudes entre l'honneur des lettrés et celui des "gens d'arme », et leurs différences fondamentales. C'était justement parce qu'ils se situaient dans deux systèmes parallèles que les lettrés et les soldats ne pouvaient jamais se rencontrer, en venir mutuellement aux mains ou se servir des armes de l'autre.

Possevino : Le lettré ne peut être dépouillé de son honneur par une injure, quelle qu'elle soit, qui lui serait faite en dehors de sa sphère de compétence.

Giberto : Et si le lettré défiait le soldat, qu'en résulterait-il ?

Possevino : Il en résulterait du blâme pour le lettré, car il se ferait connaître comme un furieux, indigne de sa profession; et il en perdrait de l'honneur plutôt que d'en gagner le moins du monde. Étant donné que nous ne sommes pas tenus de posséder tous les honneurs, qui appartiennent à toutes les activités et professions, mais seulement deux sortes d'honneur, l'honneur des mœurs et de la vie vertueuse, et l'honneur de notre profession ${ }^{14}$.

10 Cependant, le parallélisme entre les types d'honneur ne pouvait être mené trop loin. Les théoriciens rappelaient que les clercs étaient exclus du duel ${ }^{15}$ en raison d'une infériorité physique, au même titre que les femmes, les malades, les vieillards, c'est-àdire toute la catégorie des debili, mais ils soulignaient aussi que leur honneur avait plus 
de dignité, selon la hiérarchie établie dans l'Éthique à Nicomaque entre vertus actives et contemplatives ${ }^{16}$.

Car ce n'est pas honneur à un souldard d'armes de defier celuy qui faict profession toute estrange des armes: quand un armé defie un desarmé: un tout bruslé au soleil, defie celuy qui est accoustumé à l'umbre : un accoustumé de travailler et exerciter, provoque un qui est nourry à se seoir et estre oysif : un bien gras et bien nourry defie le bien maigre. Pourra le docteur à son ennemy respondre, qui le requiert ainsi. Toy qui m'appelles à combatre avec tes armes, aussi je t'appelle avec les miennes à mon camp, à sçavoir devant mes magistratz auquel lieu je monstreray avec mes armes, à sçavoir mes livres, et avec les autheurs de doctrine, combien est ton art inférieure à la mienne, et aussi ta profession ${ }^{17}$.

11 L'honneur des lettrés se réclamait de valeurs supérieures, capables de modifier les règles de l'honneur commun. C'est ainsi que les doctes se battaient pour le triomphe de la vérité, et non pour le leur propre, de sorte qu'il leur était permis plus de choses. Alors qu'un homme d'armes, par exemple, ne pouvait attaquer son professeur d'escrime sans encourir la honte, "attendu que moindre révérence n'est deue au maistre qu'au père ${ }^{18}$ », un philosophe digne de ce nom ne devait jamais se sentir arrêté par un faux respect.

Giberto : Supposons qu'un élève non seulement contredise son maître mais même écrive contre lui ; devra-t-on pour cela l'estimer ingrat et déshonoré ? [...]

Possevino: Non seulement les élèves ne perdent pas leur honneur lorsqu'ils contredisent leurs maîtres, quand ils le font pour l'amour de la vérité, mais ils le perdraient s'ils ne le faisaient pas; car c'est en la vérité seule que réside notre obligation envers nos maîtres puisque nous l'apprenons d'eux; en elle consiste notre félicité, et nous ne pourrions posséder un plus grand bien. Pour l'amour de la vérité nous sommes tenus de contredire non seulement nos maitres, mais nousmêmes, surtout quand nous sommes philosophes ${ }^{19}$.

12 S'il faut toujours garder à l'esprit cette réserve fondamentale, les analogies entre duels et disputes, honneur des armes et honneur des lettres, apparaissent fort nombreuses à qui parcourt les textes du XvI ${ }^{\mathrm{e}}$ siècle. Ces passages ou ces équivalences établis entre les deux domaines étant d'ailleurs certainement favorisés par une autre circonstance : les théoriciens italiens de l'honneur, dont les écrits se répandirent en France entre 1550 et $1580^{20}$, étaient généralement des docteurs qui tenaient à imposer leurs propres catégories. Possevino soutenait, par exemple, que les seuls experts valables en matière d'honneur et d'injures étaient les spécialistes de philosophie morale, les rois et empereurs se trouvant disqualifiés parce qu'ils ne connaissaient pas les injures ${ }^{21}$. Inversement, nombreux furent alors les gentilhommes qui embrassèrent la carrière des lettres ou des sciences, à la place de celle des armes, et qui s'évertuèrent à prouver la magnanimité de ce choix ${ }^{22}$. Je citerai seulement Ronsard, l'astronome danois Tycho Brahe, ou Galilée qui se lança avec ardeur dans la polémique à partir du moment où, échangeant son rôle de professeur contre celui d'homme de cour, il put faire brillamment état de son titre de nobil fiorentino ${ }^{23}$. Comment ne pas remarquer aussi l'insistance avec laquelle, dans ses attaques contre Érasme et dans ses efforts pour se gagner des "parrains ", Jules César Scaliger - fils d'un enlumineur padouan mais se prétendant membre de la noble famille Della Scala - étalait ses faux titres de noblesse et faisait valoir les exploits imaginaires de sa belliqueuse jeunesse ${ }^{24}$ ?

13 Si l'on abandonne les théories pour observer les comportements, l'impression n'est guère différente. Il semble avoir existé un ensemble de cadres, de formes et de codes qui entraînait certaines ressemblances, au moins superficielles, entre les manières 
combatives des différents " états », et ce quel que fût le pays. L'analogie jouait d'abord au niveau du langage. C'était une ancienne tradition que d'appliquer des termes guerriers aux disputationes (elle se profile déjà dans les Topiques), et les controversistes de la Renaissance l'illustrèrent brillamment. Or les mots déteignaient sur les usages comme le montre l'exemple des collèges jésuites, où le vocabulaire volontiers imagé du règlement s'incarnait dans une organisation quasiment militaire ${ }^{25}$.

Il existait d'ailleurs des rapports assez précis entre le protocole des joutes et des combats singuliers et celui des affrontements et querelles doctrinales. Les grandes disputes publiques prennent l'aspect de pas d'armes: l'annonce, le programme mûrement élaboré, publié et affiché, le défi lancé à la cantonade constituent autant de points de ressemblance. Pic de la Mirandole rédigea les neuf cents thèses de ses Conclusiones durant l'automne 1486 et il se rendit à Rome pour y soutenir une vaste dispute, où il aurait affronté tous ceux qui se seraient présentés; les thèses furent imprimées en décembre 1486, mais le 4 août 1487 une bulle d'Innocent VIII condamna treize thèses; les exemplaires furent détruits, la dispute n'eut jamais lieu et Pic fut arrêté à Paris. Un siècle plus tard, au printemps 1586, Giordano Bruno projeta à Paris une série de disputes publiques au collège de Cambrai, où se tenaient les cours des lecteurs royaux; le programme fut, selon la coutume, affiché à l'angle de la rue des Écoles, et Bruno fit imprimer, à ses frais, ses Cent vingt articles sur la nature et le monde contre les péripatéticiens. Lors de la première, et seule séance, Jean Hennequin présenta les thèses, puis Bruno fit une intervention fracassante, mais il fut attaqué par l'avocat Rodolphe Cailler qui s'efforça de le faire taire; l'affaire se termina par un chahut homérique et la déroute de $\mathrm{Bruno}^{26}$. La tradition des disputationes quodlibetales, à la mode parisienne, restait vivante au $\mathrm{Xv}^{\mathrm{e}}$ siècle, à Paris et en Italie, et les conflits individuels suivaient dans une certaine mesure les mêmes usages que le duel. Pour commencer, le principe de l'égalité des partenaires était partout reconnu : il fallait de puissants motifs pour qu'un supérieur acceptât d'affronter un inférieur, de sorte qu'un refus de répondre était toujours interprété comme un signe de mépris et un rappel des différences hiérarchiques. Dans sa seconde Oratio, Scaliger tirait très clairement la leçon de la querelle du Ciceronianus : les grands humanistes italiens, Alciat, Bembo et Sadolet, avaient manifesté leur supériorité en passant sous silence l'attaque d'Érasme, lequel à son tour tentait de le rabaisser lui-même en l'ignorant. "Si ces trois grands personnages, écrit-il, n'ont rien répondu à ses inepties pleines d'insolence, il m'estime au même prix, moi à qui il ne répond rien, qu'il pense avoir été lui-même estimé par eux $^{27}$.»

15 À l'université, les professeurs n'avaient pas l'habitude d'entrer eux-mêmes dans la mêlée des disputationes, ils faisaient défendre leurs thèses par les étudiants et, dans leurs rapports avec l'extérieur, ils préféraient aussi souvent faire combattre des champions à leur place. Lorsque Tartaglia, mathématicien sans chaire, reprocha violemment à Cardan, professeur de médecine à Padoue, d'avoir divulgué dans son Ars magna (1545) sa propre méthode de résolution de l'équation du troisième degré, ce fut Lodovico Ferrari, un élève de Cardan, qui lança à Tartaglia, entre février 1547 et juillet 1548, la série des six Cartelli di sfída qui l'engageaient dans un rude duel mathématique; l'affaire se conclut le 10 août 1548 par un affrontement public entre Tartaglia et Ferrari, dans l'église de Santa Maria del Giardino à Milan ${ }^{28}$. Inversement, les inconnus ou les personnes sans position qui s'en prenaient à plus hauts qu'eux étaient aussitôt soupçonnés d'ambition, à l'instar des jeunes spadassins qui lançaient des défis à tous vents. Vingt ans après l'affaire du Ciceronianus, Cardan, à son tour attaqué par 
Scaliger, faisait malicieusement état de ce précédent, en louant ironiquement «ce valeureux personnage, ce soldat d'élite qui semblait habitué à ce genre de monomachies "; il comparait son assaillant à Erostrate qui avait détruit le temple d'Ephèse, « dont il aurait été incapable d'édifier le plus petit coin », pour gagner de la renommée : «Il a entrepris de démolir notre De subtilitate dans l'espoir, à mon avis, de s'illustrer aux yeux des hommes par des disputes, où il aurait un répondant ${ }^{29}$. "

Par ailleurs, ceux qui acceptaient d'entrer officiellement en querelle en publiant contre un adversaire, c'est-à-dire en reconnaissant que celui-ci leur avait causé un tort auquel ils étaient sensibles, justifiaient souvent leur initiative par des motifs touchant à l'honneur privé même si, en réalité, la question de fond leur importait davantage. Malgré la diversité des nationalités et des contextes, il existe à cet égard quelque similitude dans le comportement de trois «maîtres ", Érasme, Cardan, et le théologien hollandais provisoirement installé à Paris, Albert Pigge. Obligés de répondre, de mauvaise grâce, à des assaillants qu'ils ne jugeaient pas de leur niveau, ils se réfèrent aux mêmes topoi et au même modèle de conduite. Tous trois se présentent comme des hommes de silence et d'études, qui ont mieux à faire qu'à se disperser dans de vaines querelles sophistiques. Érasme, dans sa Réponse au Plaidoyer pour l'Italie de Pietro Corsi, affirme sa volonté initiale de ne rien répondre, de ne pas seulement daigner lire une attaque qui le vise sans l'atteindre, et rappelle son dégoût constant pour ces emportements furieux, indignes de serviteurs des Muses, fussent-ils païens ${ }^{30}$. Cardan dresse, à l'intention de Scaliger, un parallèle accablant entre les belles sciences de la nature et les disciplines sans objet propre, comme la dialectique qui, poussées trop loin, ne produisent qu'orgueil gonflé de vide, surtout mises au service de matamores hargneux ${ }^{31}$. Quant à Pigge, traité de cacastrologus par Marco da Benevento qui n'acceptait pas sa théorie de la huitième sphère, il s'étonne avec une ironie amère qu'un vieillard, un prêtre, un théologien, un moine (Marco possédait toutes ces qualités) n'eût pas rougi de l'entraîner dans ce combat déshonorant: «Si les affrontements humains ne sont pas absolument sans fruit, lorsqu'ils sont menés par amour pour la recherche de la vérité, sans tomber dans la rage, quand le conflit s'enflamme à ce point, en quoi le spectacle est-il différent de celui qu'offrent des bonnes femmes qui en viennent aux mains, ou un bouffon qui en insulte un autre ${ }^{32}$ ?"

Les « maîtres » tiennent donc un langage conforme à leur statut qui doit les garder audessus de la mêlée et les dispenser d'obéir aux consignes du système de l'honneur ordinaire. Cardan, notamment, prend un malin plaisir à disqualifier les valeurs prônées par Scaliger : il affirme qu'il préfère perdre de la gloire plutôt que du temps; il se moque des prétentions nobiliaires de son assaillant - «ce que nous n'avons pas fait nous-même est à peine à nous »-, et conclut : "Qu'il soit d'une souche aussi illustre qu'il veut, et qu'il me suffise à moi d'être Hieronymus Cardanus ${ }^{33}$ ».

18 Et pourtant, disent-ils, des motifs d'ordre non scientifique les forcent à rompre le silence pour éviter plus grand scandale. Marco da Benevento a publié sans autorisation une lettre d'Albert Pigge à Federico Carafa, compromettant ainsi ce prince ${ }^{34}$; des ennemis romains d'Érasme ont affiché dans les rues une lettre écrite à des amis et monté une machination, incluant la fabrication d'un faux, pour jeter de l'huile sur le $\mathrm{feu}^{35}$; Scaliger, qui ne connaît pas Cardan, s'est permis des critiques personnelles, que ses proches n'avaient jamais songé à formuler, et avait même mis en cause ses convictions religieuses, se conduisant ainsi en violentissimus inimicus et en vil calomniateur, non pas en opposant philosophique ${ }^{36}$. Cette tendance à ne pas 
"brancher" directement l'affrontement sur la question de fond (la bravoure des Italiens, la vérité des thèses du De subtilitate ou de la théorie de la huitième sphère) pourrait faire songer, toutes proportions gardées, à l'usage des duels dans lesquels on ne se battait pas pour l'injure première, mais pour l'injure seconde que constituait le démenti ${ }^{37}$.

Un autre élément de rapprochement pourrait concerner l'appel aux "parrains ». Les dédicataires de nos textes de controverses jouent en effet un rôle particulier; ils ne sont pas les bénéficiaires d'un simple hommage, on leur demande de jouer le rôle d'experts et d'arbitres, à la manière des moderatores des disputes universitaires ou encore des «parrains » qui assistaient les duellistes : «Il y hà des Maistres, ou de vray dire advocatz des combatans, qu'on appelle Parrains, de grand estime en l'art de guerre, et qui font profession de la science des armes. Est bien nécessaire les combatans de se aider de ceulx cy, entant que s'il survient aucun différent ou doubte, oultre la contention des combatans, le déterminent entre eulx mesmes ${ }^{38}$."

Albert Pigge en appelle au patronage (ou au parrainage ?) d'Agostino Nifo, «Prince des philosophes de notre âge ${ }^{39}$ "; Érasme s'en remet au jugement de Johann Koler, pour qu'il décide de l'usage qu'il convient de faire de sa Responsio ${ }^{40}$. Que dire de la façon dont Scaliger, au moment de sa première Oratio contre le Ciceronianus, bombarde de lettres les élèves des collèges parisiens, le recteur de l'Université et Noël Beda, syndic de la Faculté de théologie et notoire ennemi d'Érasme, afin de les contraindre, bon gré mal gré, à lui servir de moderatores ou de judices, selon ses propres termes ${ }^{41}$ ?

Cette enquête très limitée met en évidence l'existence d'un savoir-vivre européen de la dispute savante. Les particularismes nationaux existaient sans doute, mais ne me semblent avoir joué qu'un rôle superficiel: tout le monde applique à peu près les mêmes règles, ce qui permet d'interpréter correctement le comportement d'un adversaire étranger; et l'habitude de reprocher à cet adversaire les défauts soi-disant propres à sa nation relève du lieu commun. Peut-être doit-on discerner une certaine influence de la mode des duels sur le comportement des savants d'Italie ${ }^{42}$, remarquer que ce pays a produit les plus ardents provocateurs au début du XvI ${ }^{\mathrm{e}}$ siècle, et comparer dans cette perspective la conduite vibrionnante d'un Scaliger et la relative pondération d'un Érasme. Mais je doute que ces observations mènent bien loin.

Une fois posée l'homogénéité d'un comportement et d'une éthique, il s'agit de réfléchir à leurs fondements. À mon avis, leur socle le plus solide était constitué par la tradition universitaire, par les modes de discussions qu'elle avait instaurés, et par le système de valeurs, à la fois morales et sociales, qu'elle reconnaissait. Le discours humaniste s'était surimposé à cette tradition, il l'avait enrichi plus qu'il ne l'avait foncièrement mise en cause. Quand aux analogies avec le système parallèle des querelles aristocratiques, je ne songerais pas à en nier l'importance mais en prenant bien soin de ne jamais confondre le plan des métaphores et celui de la causalité.

Il vaudrait aussi sûrement la peine de chercher s'il a existé une évolution sensible entre la fin $\mathrm{du} \mathrm{Xv}^{\mathrm{e}}$ et le $\mathrm{XVII}^{\mathrm{e}}$ siècle, moment où nombre de savants quittèrent le milieu des universités pour celui des cours et où le progrès des régimes absolutistes perturba sensiblement le système de l'honneur ${ }^{43}$, mais je laisserai la question ouverte. 


\section{NOTES}

1. Cf. R. Barber et J. Barber, Les Tournois, Paris, Cie 12, 1989 (éd. originale: Woodbridge, The Boydell Press, 1989), p. 117 sq.; E. Fournier, Le Jeu de paume, son histoire et sa description, Paris, Didier, 1862 ; J. Marshall, The Annals of Tennis, Londres, "The Field» Office, 1878 ; A. de Luze, La magnifique histoire du jeu de paume, Bordeaux, Delmas, 1933. Sur la valeur modélisante de ce jeu, qui autorise une comparaison au moins partielle avec la querelle savante, cf. M.-M. Fontaine, Libertés et savoirs du corps à la Renaissance, Caen, Paradigme, 1993, p. 99-111.

2. Selon Diogène Laerce (Vies et opinions des philosophes, IX, 52), Protagoras aurait été le premier à organiser des joutes où s'affrontaient les tenants de thèses contraires ; cf. Platon, Sophiste, 232 be. Sur l'éristique aristotélicienne, cf. P. Moraux, «La joute dialectique d'après le huitième livre des Topiques ", in G. E. L. Owen, ed., Aristotle on dialectic. The Topics, Oxford, Clarendon Press, 1968, p. 277-311.

3. Cf. notamment J. L. Vivès, De disciplinis libri XX, Anvers, M. Hillenius, 1531, I, « De corruptis artibus ", notamment aux ff. 13 v. sq. ; III, « De disputatione », f. 73 v. sq.

4. J. L. Vivès, De disciplinis libri XX, ibid., II, « De tradendis disciplinis », f. 101 v.

5. P. Ramus, Advertissement sur la reforme de l'université de Paris, Paris, Wechel, 1562.

6. Saint Ignace avait recommandé dans ses Constitutions la pratique assidue de la dispute, et l'idée fut reprise par les rédacteurs des versions successives de la Ratio studiorum. Cf. notamment le règlement des disputationes dans la version de 1586 - G. M. Pachtler, ed., Ratio studiorum [...] S.J. per Germaniam olim vigentes, II, Berlin, 1887, p.100-107. Cf. aussi F. de Dainville, Les Jésuites et l'éducation de la société française. La naissance de l'humanisme moderne, Paris, Beauchesne, 1940, I, p. 136 sq. Le règlement du collège de La Flèche, fondé en 1603, réservait aux disputationes un rôle important. Les élèves de philosophie s'y livraient un dimanche soir sur trois au réfectoire: «Durant le premier quart d'heure [les philosophes] devaient donner en orateurs une idée juste, claire et amplifiée de la question qui faisait le sujet de la dispute. Le second quart d'heure devait être employé à prouver solidement leur opinion, et le troisième était pour répondre aux objections faites par ceux qui composaient l'assemblée.» («Règlement de MM.les pensionnaires ", $2^{\mathrm{e}}$ partie, 12 , cité dans C. de Rochemonteix, Un collège de jésuites aux XVII et XVIII siècles : Le collège Henri IV de La Flèche, Le Mans, Leguicheux, 1889, II, p. 35.) Lors des fêtes pour la canonisation de saint Ignace, en juillet 1622, les disputes firent partie des spectacles offerts au public. Le mardi matin eut lieu une grande dispute théologique sur la grâce, le mercredi et le jeudi des séances de joutes philosophiques dans la classe concernée : «Les auditeurs interrogent, discutent. Tout se fait en latin, les thèses sont imprimées sur satin grand in-4.» (Ibid., II, p. 148-153.)

7. J. L. Vivès, De disciplinis libri XX, op. cit., «De corruptis artibus », f. 13 v.-14 r. Cf. aussi le « De tradendis disciplinis ", f. 101 v., qui renvoie au chapitre XI de l'Épitre aux Romains.

8. Traduction par F. de Dainville, Les Jésuites et l'éducation de la société française..., op. cit., p. 137.

9. M. de Montaigne, Essais, III, 8 (éd. Villey-Saulnier, Paris, Presses universitaires de France, 1978), II, p. 923. Ce qui précède montre que Montaigne songeait à de véritables débats organisés, à la manière des disputationes, et non pas à la simple conversation comme l'interprète Villey : "Les Athéniens, et encore les Romains, conservoient en grand honneur cet exercice en leurs Académies. De nostre temps, les Italiens en retiennent quelques vestiges, à leur grand profict, comme il se voit par la comparaison de nos entendemens aux leurs. » (p. 922-923.)

10. Dans l'avis au lecteur, qui commente la fausse nouvelle de la mort de Cardan, Scaliger se plaint d'avoir perdu la source, l'aliment et même l'«auteur» de ses études et recherches: Exotericarum exercitationum..., Paris, F. Morel, 1557, f. 2 v.

11. Ibid., f. 476 v. 
12. Sur l'adaptation des catégories morales à la diversité des états, voir notamment A. Jouanna, «Recherches sur la notion d'honneur au XVI ${ }^{\mathrm{e}}$ siècle ", Revue d'Histoire moderne et contemporaine, XV, 1968, p. 596-623.

13. Notons pourtant qu'il existait au moins deux lectures de l'Éthique au $\mathrm{XvI}^{\mathrm{e}}$ siècle, la lecture littérale et la lecture christianisée, représentée par la très célèbre Introductio in Ethicen de Lefèvre d'Etaples commentée par Josse Clichtove. La magnanimité, pour la première, c'était de savoir requérir les grands honneurs dont on se sait digne (voir par exemple J. Bouchereau, Flores Aristotelis, Paris, Marnef, 1563, f. 180 r., qui se réfère à Ethica, IV, ch. 3) ; pour la seconde c'était, en dernier ressort, l'humilité et la patience accomplies dont la Vierge Marie et Job avaient donné le modèle (In Ethicen introductio, Paris, Colines, 1537, f. 18 r.). Tandis qu'un G. B. Possevino, partisan du juste duel, se situait dans la première ligne interprétative, Giulio Landi inséra son plaidoyer contre les combats singuliers dans la paraphrase dialoguée qu'il réalisa de l'lntroductio de Lefèvre et Clichtove (Le attioni morali..., Venise, Giolito, 1564). Cf. E. Garin, « Echi italiani di Erasmo e di Lefevre d'Etaples », Rivista critica di Storia della Filosofia, XXVI, 1971, p. 88-90.

14. G. B. Possevino, Dialogo dell'honore, Venise, Giolito, 1553, p. 128-129.

15. A. Alciat, De singulari certamine seu duello tractatus, Paris, Kerver, 1541, f. 38 v.-39 r.

16. Cf. G. B. Possevino, Dialogo dell'honore, op. cit., p. 188-189.

17. Le livre du duel et combat singulier, faict par le Sr André Alciat, jurisconsulte tres excellent, traduit de latin en françoys par I.D.L.F., Paris, J. André, 1550, f. 25 v.

18. A. Alciat, Le livre du duel, op. cit., f. $31 \mathrm{v}$.

19. G. B. Possevino, Dialogo dell'honore, op. cit., p. 156. L'exemple avancé est celui, classique, de l'attaque menée par Aristote contre la doctrine de Platon.

20. Cf. notamment F. Erspamer, La biblioteca di Don Ferrante. Duello e onore nella cultura del Cinquecento, Rome, Bulzoni, 1982; F. Billacois, Le duel dans la société française des XVI ${ }^{e}-\mathrm{XVII}{ }^{e}$ siècles, Paris, Éd. de l'EHESS, 1986.

21. G. B. Possevino, Dialogo dell'honore, op. cit., p. 305-306.

22. Cf. M. Fumaroli, «Éthique et rhétorique du héros humaniste. Du magnanime à l'homme de ressentiment ", Papers on French XVIIth Century Literature, IV-V, 1976, p. 167-201, notamment p. 172 : la Renaissance «atténue l'antithèse médiévale entre le chevalier et le clerc. L'héroïsme humaniste englobe tous les idéaux spécialisés ».

23. Cf. M. Biagioli, Galileo Courtier. The Practice of Science in the Culture of Absolutism, Chicago, Chicago University Press, 1993. Sa comparaison fort stimulante entre duels et disputes scientifiques (p. 60-73) ne distingue peut-être pas assez clairement le duel aristocratique et les affrontements réglés se déroulant sous l'autorité de princes mécènes dans les cours du XVII ${ }^{\mathrm{e}}$ siècle : à cette époque, le duel était devenu un acte d'insubordination. Par ailleurs, elle met au second plan l'enjeu de vérité dans les querelles menées par Galilée qui se sentait philosophe autant que courtisan et défendait son honneur suivant les lois propres à cette sphère de compétence (voir les textes de Possevino cités supra).

24. Cf. par exemple la première lettre de protestation adressée aux collèges parisiens auxquels il avait envoyé le manuscrit de son Oratio pro Cicerone, et qui n'avaient pas daigné répondre : J. C. Scaliger, Epistolae aliquot nunc primum vulgatae [éd. de Ph.-J. de Maussac], Toulouse, Bosc et Colomies, 1620, p. 8. Cf. J. F. C. Richards, "The Elysium of Julius Caesar Bordonius ", Studies in the Renaissance, IX, 1962, p. 195-217 ; M. Billanovitch, « Benedetto Bordon e Giulio Cesare Scaligero », Italia medioevale e umanistica, XI, 1968, p. 187-256.

25. Cf. le chapitre vi de la Ratio studiorum de 1586, éd. Pachtler, p. 170 sq.; voir aussi F. de Dainville, Les Jésuites et l'éducation de la société française..., op. cit., p. 142 sq. (« Des écoliers piqués de gloire »).

26. A. Perfetti, « Un nuovo documento sul secondo soggiorno parigino di G. B. », in E. Canone, ed., G. Bruno : Gli anni napoletani e la peregrinatio europea, Cassin, 1992, p. 99-109.

27. J. C. Scaliger, Contra Desid. Erasmum Roterodamum oratio II..., p. 23. 
28. A. Masotti, « Sui Cartelli di matematica disfida scambiati fra L. Ferrari e N. Tartaglia », Rendiconti dell'Istituto lombardo di Scienze e Lettere, 94, 1960, p. 31-41; A. Masotti, Studi su N. Tartaglia, Brescia, 1962.

29. J. Cardan, In calumniatorem librorum de subtilitate actio (à la suite de Quaedam opuscula, Bâle, H. Petri, 1559, p.142, 137, 138). La même comparaison avec le temple d'Ephèse, à double tranchant, était venue à Scaliger, lors de sa première attaque contre Érasme (Epistolae aliquot nunc primum vulgatae, op. cit., p. 21).

30. D. Érasme, Responsio ad Petri Cursii defensionem nullo adversario bellacem, Bâle, Froben, 1535, dédicace à Johann Koler, f. a2 r., p. 31. La Defensio pro Italia ad Erasmum de P. Corsi, dédiée à Paul III (Rome, 1535), réfutait quelques passages d'Érasme qui mettaient en doute la bravoure des Italiens.

31. J. Cardan, In calumniatorem librorum de subtilitate actio, op. cit., p. 139-140.

32. A. Pigge, Adversus novam Marci Beneventani astronomiam [...] Apologia, Paris, Colines, 1522, f. a1 r-v. Cardan s'indignait aussi de ce que Scaliger, vieillard et philosophe, s'amusât à ces attaques ineptes (op. cit., p. 142).

33. J. Cardan, In calumniatorem librorum de subtilitate actio, op. cit., p. 142-143.

34. A. Pigge, Adversus novam Marci Beneventani astronomiam, op. cit., f. A4 r.-v.; a1 r.

35. D. Érasme, Responsio, op. cit., p. 3-4, 29-30.

36. J. Cardan, In calumniatorem librorum de subtilitate actio, op. cit., p. 138-139.

37. Sur l'importance du démenti (la mentità italienne) dans le déclenchement du duel, voir notamment H. Morel, «La fin du duel judiciaire en France et la naissance du point d'honneur », Revue historique de Droit français et étranger, $4^{\text {e }}$ s., 42, 1964, p. 574-639 ; G. B. Possevino, Dialogo dell'honore, op. cit., p. 281, 284, etc. Il apparaît bien chez Possevino que le partage des rôles se faisait autour de la mentità, entre celui qui « est démenti » (attore) et est donc injurié, et celui qui donne le démenti (reo) (p. 244). À la fin du Xvi ${ }^{\mathrm{e}}$ siècle des voix s'élevèrent, soit pour s'étonner de la puissance accordée au « démentir » (Montaigne, Essais, II, 18, éd. Villey, p. 666-667), soit pour s'en indigner (G. Landi, op. cit., p. 141, 221-222, etc.).

38. A. Alciat, Le livre du duel, op. cit., f. 43 r.

39. A. Pigge, Adversus novam Marci Beneventani astronomiam, op. cit., f. A2 r., A4 v.-A6 r. Agostino Nifo était un connaisseur en matière de duel verbal. Lors de la controverse suscitée par le De immortalitate animae de Pomponazzi, il pria ainsi Léon X de lui accorder le champ : A. Nifo, De immortalitate animae libellus, Venise, 1518, dédié au pape, cité dans F. Fiorentino, Pietro Pomponazzi, Florence, Le Monnier, 1868, p. 52.

40. D. Érasme, Responsio, op. cit., p. 31.

41. J. C. Scaliger, Epistolae aliquot nunc primum vulgatae, op. cit., p. 7-21.

42. M. Biagioli, Galileo Courtier..., op. cit., p. 23, a noté que Girolamo Muzio, fameux théoricien du duel, fait partie des témoins qui signent le premier Cartello envoyé à Tartaglia.

43. M. Biagioli a amorcé ce type d'enquête, mais il ne distingue pas les différents régimes de l'honneur (celui des intellectuels, celui des aristocrates) et recherche surtout les similitudes : il rapproche ainsi la querelle Tartaglia-Ferrari-Cardan (tout à fait étrangère à la cour) et les querelles menées par Galilée sous le patronage du grand-duc ou des Lincei (ibid., p. 61-62). Il ne remarque pas à quel point il pouvait être paradoxal qu'un « courtisan » comme Galilée attachât autant de prix à défendre son honneur personnel et à proclamer sa virtù, dans ce qu'il appelle « la culture de l'absolutisme ». 


\section{RÉSUMÉS}

À la Renaissance et au début de l'époque moderne, grâce au développement de l'imprimerie, la querelle savante acquiert une dimension internationale et un caractère largement public. L'analyse de quelques cas, pris à des domaines divers, révèle l'existence d'un savoir-vivre international de la querelle, fondé sur la reconnaissance de valeurs éthiques communes. La plupart des « intellectuels » d'Europe ont alors reçu une formation similaire qui les a familiarisés avec les règles de la «dispute» et leur a fait partager la croyance en son utilité dans la constitution du savoir. Plus généralement encore, les querelles sont pour eux l'occasion de manifester leur participation au système général de l'honneur, tel qu'il est défini dans l'Éthique d'Aristote. L'honneur des clercs et des professeurs se distingue de celui des « gens d'armes », tout en se développant sur un plan parallèle, et il est assez universellement reconnu pour garantir l'homogénéité et l'intelligibilité des comportements querelleurs.

At the Renaissance and in the beginning of the modern period, thanks to the development of the printing press, the scholarly quarrel acquired an international dimension and a largely public character. The analysis of some examples taken from different domains reveals the existence of an international ethics of the quarrel, based on the recognition of common ethical values. Most of Europe's « intellectuals " then had received a similar training which had familiarised them with the rules of the "dispute" and made them share the belief in its service to the constitution of knowledge. Yet more generally, the quarrels were for them an occasion to manifest their participation in a general system of honour such as it was defined in Aristotle's Ethic. The honour of clerics and professors was distinguished from that of "men of arms" even while developing on a parallel plane, and it was sufficiently universally recognised to guarantee the homogeneity and intelligibility of the quarellers' behaviour.

\section{AUTEUR}

\section{ISABELLE PANTIN}

Isabelle Pantin (Université du Maine, Le Mans) enseigne la littérature des XVI ${ }^{\mathrm{e}}$ et XVII ${ }^{\mathrm{e}}$ siècles. Ses recherches portent sur l'histoire intellectuelle, surtout dans le domaine de la cosmologie. Elle a récemment publié des éditions et traductions de textes de Galilée et de Kepler (Paris, Les Belles Lettres, 1992 et 1993), et un livre sur La Poésie du ciel en France dans la seconde moitié du XVI ${ }^{e}$ siècle (Genève, Droz, 1995). 